\title{
APPLYING DEVICE SIMULATION FOR LIFETIME-CONTROLLED DEVICES
}

\author{
R.Siemieniec ${ }^{1}$, W.Südkamp ${ }^{2}$, J.Lutz ${ }^{3}$ \\ ${ }^{1}$ Ilmenau Technical University, PO Box 100565, D-98684 Ilmenau, Germany, e-mail: ralf.siemieniec@tu-ilmenau.de \\ ${ }^{2}$ Aktiv Sensor GmbH, Ruhlsdorfer Str.95, D-14532 Stahnsdorf, Germany, e-mail: wsuedkamp@aktiv-sensor.de \\ ${ }^{3}$ Technical University of Chemnitz, D-09107 Chemnitz, e-mail: josef.lutz@infotech.tu-chemnitz.de
}

\begin{abstract}
Irradiation techniques are widely used for carrier lifetime control in power devices. Improvements of irradiated devices were usually realized by a number of experiments. The use of an extended recombination model allows improved device simulations which explain the temperature dependencies of stationary and dynamical characteristics. Due to that progress device simulation is able to support development and optimization of irradiated devices.
\end{abstract}

\section{INTRODUCTION}

Lifetime killing is usually used for optimization of power device characteristics because of the very good reproducibility. By applying irradiation (e.g. with electrons, with protons or with alpha particles), it is possible to generate homogenous or local lifetime profiles as well which is another advantage compared to the well-known recombination centers gold and platinum [1]. Furthermore, the temperature dependencies of irradiation-induced centers are different than by using platinum or gold [2] which results in different temperature-dependent properties of the devices. To explain the behavior of irradiated devices it is necessary to consider a number of recombination centers since different centers may control device characteristics under different conditions.

Based on DLTS (Deep Level Transient Spectroscopy) and lifetime measurements [3,4], the center properties of the most important traps after electron or helium irradiation and annealing with a temperature above $300^{\circ} \mathrm{C}$ have been determined in previous work $[5,6]$.

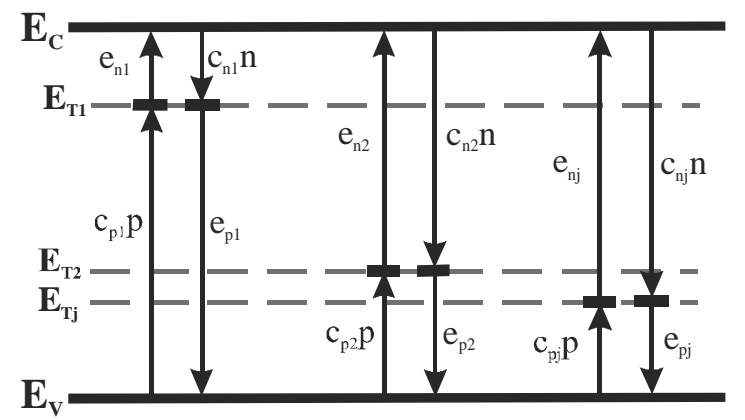

Fig.1: Recombination via a number of independent recombination centers
Introducing these parameters into simulation and the use of an extended recombination model explains static and dynamic device characteristics in a wide temperature range.

\section{RECOMBINATION MODEL}

Irradiation generates centers with different energy levels in the band gap of Silicon semiconductors. Each level may act as an effective recombination center where the total recombination rate results from the emission and capture processes of each single level as illustrated in figure 1 . Thus an advanced recombination model is used which includes full trap dynamics of a number of independent recombination centers as fully described in [6]. This model allows the simulation of the complete dynamical behavior of a device with more than one recombination level under different conditions and considers the trap charging processes as well.

For all simulations, the 2D device simulator TeSCA is used [7]. TeSCA solves the three fundamental equations - the Poisson equation and the electron and hole current continuity equation. For considering deep traps, these equations have to be extended. In the Poisson equation (1), the charged recombination centers have to be considered:

$$
-\operatorname{div}(\varepsilon \cdot \operatorname{grad} \varphi)=q\left[p-n-N_{A}^{-}+N_{D}^{+}+\sum\left(N_{T D}^{+}-N_{T A}^{-}\right)\right]
$$

In the continuity equations, the thermal capture and emission processes of carriers via the deep levels within the band gap lead to additional recombination terms as shown in equations (2) and (3). The occupancies of the acceptor or donator traps are evaluated from the balance equations (4) and (5).

$$
\begin{aligned}
& \frac{\partial n}{\partial t}-\operatorname{div} J_{n}=G- R+\sum\left[e_{n A} N_{T A}^{-}-c_{n A} n\left(N_{T A}-N_{T A}^{-}\right)\right] \\
&+ \sum\left[e_{n D}\left(N_{T D}-N_{T D}^{+}\right)-c_{n D} n N_{T D}^{+}\right] \\
& \frac{\partial p}{\partial t}+\operatorname{div} J_{p}=G- R+\sum\left[e_{p A}\left(N_{T A}-N_{T A}^{-}\right)-c_{p A} p N_{T A}^{-}\right] \\
&+\sum\left[e_{p D} N_{T D}^{+}-c_{p D} p\left(N_{T D}-N_{T D}^{+}\right)\right] \\
& \frac{d f_{A}}{d t}=\left(c_{n A} n+e_{p A}\right)\left(1-f_{A}\right)-\left(c_{p A} p+e_{n A}\right) f_{A}
\end{aligned}
$$


TABLE II

Sample type overview

\begin{tabular}{c|ccc|c} 
Sample & \multicolumn{3}{|c|}{ Irradiation } & pn-junction \\
& Type & Energy & Dose & depth \\
\hline $\mathrm{N}$ & none & - & - & $11.5 \mu \mathrm{m}$ \\
\hline $\mathrm{H} 1$ & Helium & $5.4 \mathrm{MeV}$ & $10 \%$ & $11.5 \mu \mathrm{m}$ \\
$\mathrm{H} 2$ & Helium & $5.4 \mathrm{MeV}$ & $100 \%$ & $11.5 \mu \mathrm{m}$ \\
\hline $11 \mathrm{E} 1$ & Electron & $1.1 \mathrm{MeV}$ & $10 \%$ & $22 \mu \mathrm{m}$ \\
$11 \mathrm{E} 2$ & Electron & $1.1 \mathrm{MeV}$ & $50 \%$ & $22 \mu \mathrm{m}$ \\
$11 \mathrm{E} 3$ & Electron & $1.1 \mathrm{MeV}$ & $100 \%$ & $22 \mu \mathrm{m}$ \\
\hline $100 \mathrm{E} 1$ & Electron & $10 \mathrm{MeV}$ & $3 \%$ & $22 \mu \mathrm{m}$ \\
$100 \mathrm{E} 2$ & Electron & $10 \mathrm{MeV}$ & $6 \%$ & $22 \mu \mathrm{m}$ \\
$100 \mathrm{E} 3$ & Electron & $10 \mathrm{MeV}$ & $10 \%$ & $22 \mu \mathrm{m}$ \\
\hline $\mathrm{EH}$ & Electron & $1.1 \mathrm{MeV}$ & $100 \%$ & $22 \mu \mathrm{m}$
\end{tabular}

$\frac{d f_{D}}{d t}=\left(c_{p D} p+e_{n D}\right)\left(1-f_{D}\right)-\left(c_{n D} n+e_{p D}\right) f_{D}$

\section{SAMPLE PREPARATION}

Samples were prepared using the Semikron CAL-diode (CAL: Controlled Axial Lifetime) production line. All devices have an active area of $6 \mathrm{~mm}^{2}$ and were annealed at over $300^{\circ} \mathrm{C}$ for one hour. Sample EH contains the most important lifetime adjustment steps of the CALdiode. In order to perform DLTS measurements, the junction depth of the Helium only radiated devices was reduced as shown in Table II. Furthermore, in Sample $\mathrm{H} 1$, the Helium dose was reduced to $10 \%$ of sample EH to allow the evaluation of the electric parameters of the recombination centers. The rated current is about $10 \mathrm{~A}$ $\left(166 \mathrm{~A} / \mathrm{cm}^{2}\right)$, the rated voltage is $1200 \mathrm{~V}$.

\section{RECOMBINATION CENTER PROPERTIES}

Table I shows the center properties as used in the simulations according to previous work [6]. In comparison to the work in [6], the estimation of the electron capture rate of $\mathrm{E}(90 \mathrm{~K})$ was widened to samples

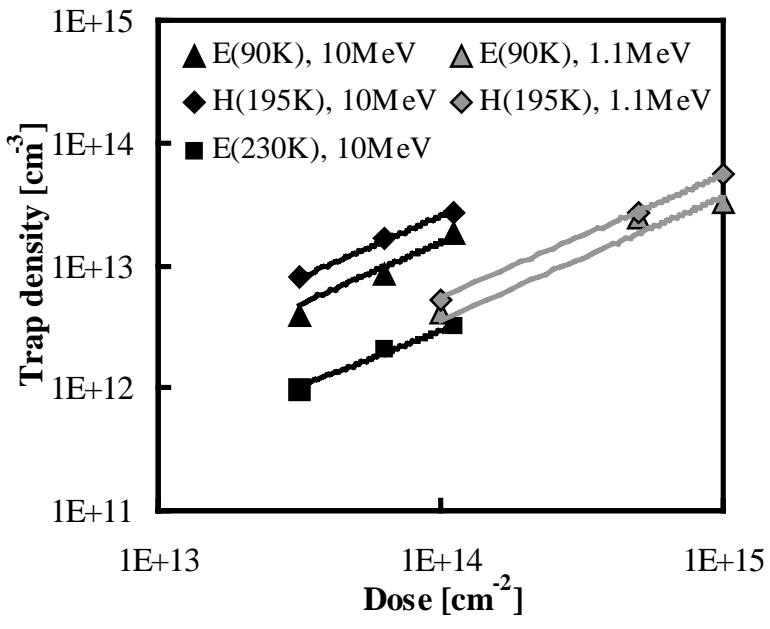

Figure 2: Irradiation dose dependent trap density

with an electron irradiation energy of $10 \mathrm{MeV}$ as shown in Table II. Therefore, the value of this electron capture rate shows some small derivations compared to [6] which are within the error limits of the measurement techniques.

The concentration dependence of the generated recombination centers on irradiation dose is approximately linear as shown in figure 2 . The trap $\mathrm{E}(230 \mathrm{~K})$ is not found in the $1.1 \mathrm{MeV}$ electron irradiated samples due to the annealing process.

DLTS measurements were also used for the determination of the concentration profiles in the helium radiated samples. Due to the high concentrations, only the profile of $\mathrm{E}(230 \mathrm{~K})$ in sample $\mathrm{H} 1$ was detectable. For the traps $\mathrm{H}(195 \mathrm{~K})$ and $\mathrm{E}(90 \mathrm{~K})$ the same profile is assumed and the peak concentrations are approximated from the amplitude of the DLTS signal compared to the DLTS signal of E(230K). For an approximation of the profiles in sample $\mathrm{H} 2$, lifetime measurements were used for the estimation of the highinjection lifetime. The high-injection lifetime represents an average lifetime depending on the recombination center maximum [8].

TABLE I:

Recombination center properties

\begin{tabular}{c|c|c|c} 
Trap & Energy level & $\mathrm{c}_{\mathrm{n}}\left[\mathrm{cm}^{3} / \mathrm{s}\right]$ & $\mathrm{c}_{\mathrm{p}}\left[\mathrm{cm}^{3} / \mathrm{s}\right]$ \\
\hline $\mathrm{E}(90 \mathrm{~K})$ & $\mathrm{E}_{\mathrm{C}-\mathrm{E}_{\mathrm{T}}=0.167 \mathrm{eV}}$ & $1.15 \cdot 10^{-7} \exp \left(-\frac{T}{355.4 K}\right)$ & $6.39 \cdot 10^{-7} \sqrt{\frac{T}{300}} \exp \left(\frac{6.15 \cdot 10^{-3}}{k_{B} T}\right)$ \\
$\mathrm{E}(230 \mathrm{~K})$ & $\mathrm{E}_{\mathrm{C}}-\mathrm{E}_{\mathrm{T}}=0.447 \mathrm{eV}$ & $3.41 \cdot 10^{-8} \sqrt{\frac{T}{300}} \exp \left(\frac{22.13 \cdot 10^{-3}}{k_{B} T}\right)$ & $2.79 \cdot 10^{-8} \sqrt{\frac{T}{300}} \exp \left(-\frac{22.13 \cdot 10^{-3}}{k_{B} T}\right)$ \\
$\mathrm{H}(195 \mathrm{~K})$ & $\mathrm{E}_{\mathrm{T}-\mathrm{E}_{\mathrm{V}}=0.351 \mathrm{eV}}$ & $9.85 \cdot 10^{-9} \sqrt{\frac{T}{300}} \exp \left(-\frac{85 \cdot 10^{-3}}{k_{B} T}\right)$ & $4.3 \cdot 10^{-9} \sqrt{\frac{T}{300}}$
\end{tabular}



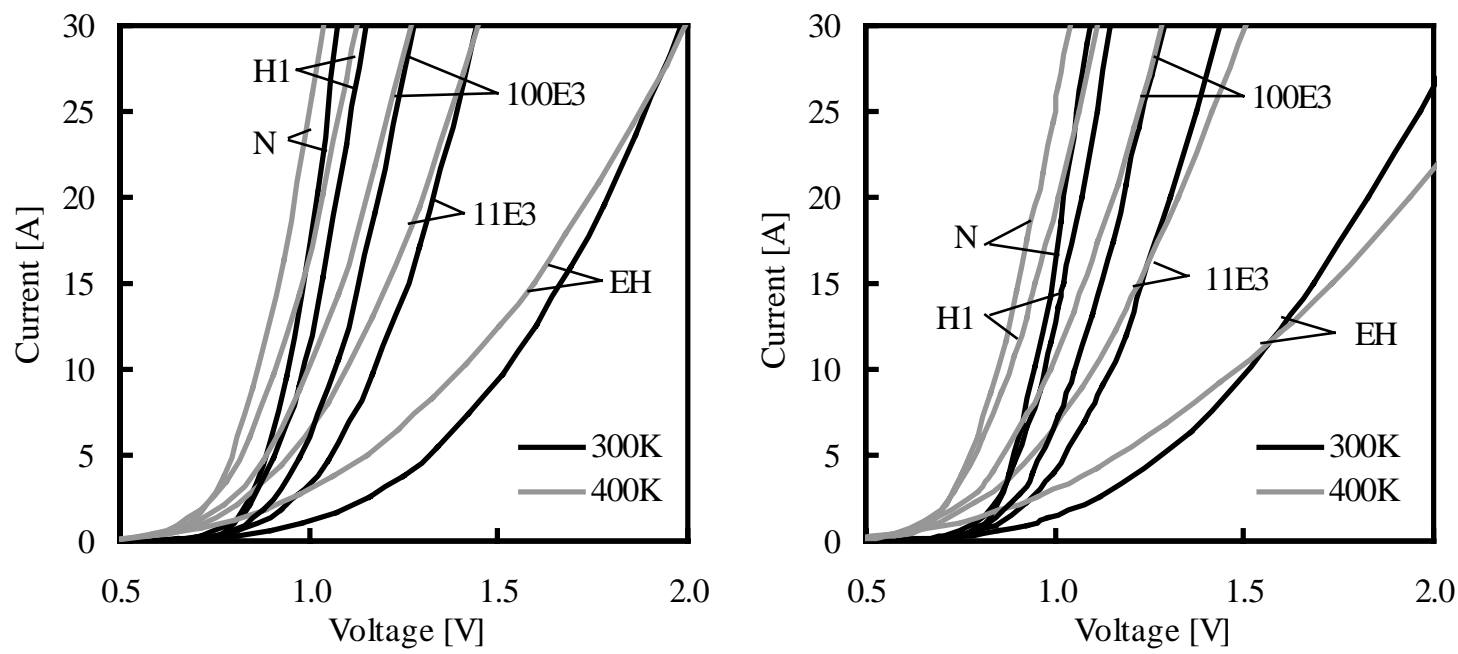

Figure 3: Comparison of forward voltage for different samples in simulation (left) and experiment (right)

\section{APPLICATION POSSIBILITIES}

First the forward voltage behavior is investigated. Figure 3 shows simulated and measured characteristics for different samples at temperatures of $300 \mathrm{~K}$ and 400K. Since series resistances such as bonding wires influences the forward characteristics, these resistances and their temperature dependencies were measured and further eliminated in the measurement results.

The comparison between measurements and simulations shows good accordance for the non- and electron-irradiated samples while we found some discrepancies at the helium and the helium- and electron-irradiated devices. This might be caused by the uncertainties in the concentration profile estimation as stated before. Here, further efforts in the concentration profile determination are necessary.

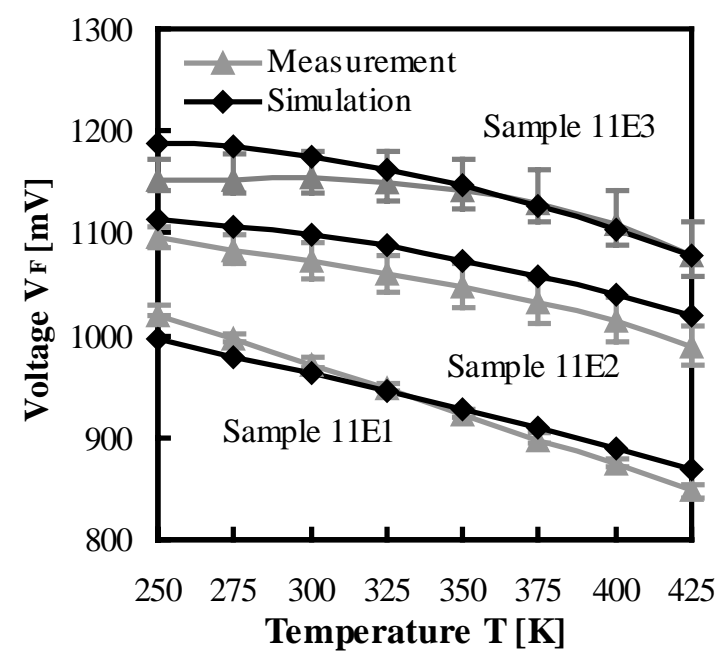

From further interest are the results presented in figure 4. Here, the forward voltage dependency of the electron-irradiated samples on temperature at nominal current is shown. The good accordance of simulations and measurements gives evidence about correctly estimated parameters of the dominant center $\mathrm{E}(90 \mathrm{~K})$.

Using simulations, reverse recovery behavior is studied and compared to measurements. Figure 5 shows the reverse recovery waveforms for different samples. Again a good accordance of simulated and measured waveforms is found. Furthermore, figure 5 shows the consequences of the application of different irradiation types. Obviously, the combination of electron and helium irradiation leads to the lowest turn-off losses. Figure 6 compares the stored charge in simulation and experiment for different samples. The dependencies on irradiation type and temperature are correctly calculated

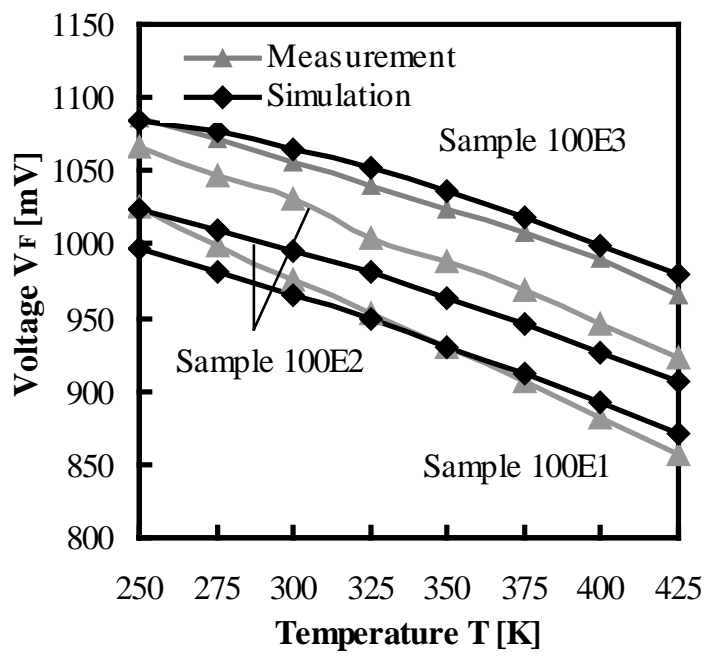

Figure 4: Temperature dependence of forward voltage at nominal current $\mathrm{I}_{\mathrm{F}}=10 \mathrm{~A}\left(\mathrm{~J}_{\mathrm{F}}=166 \mathrm{~A} / \mathrm{cm}^{2}\right)$ 

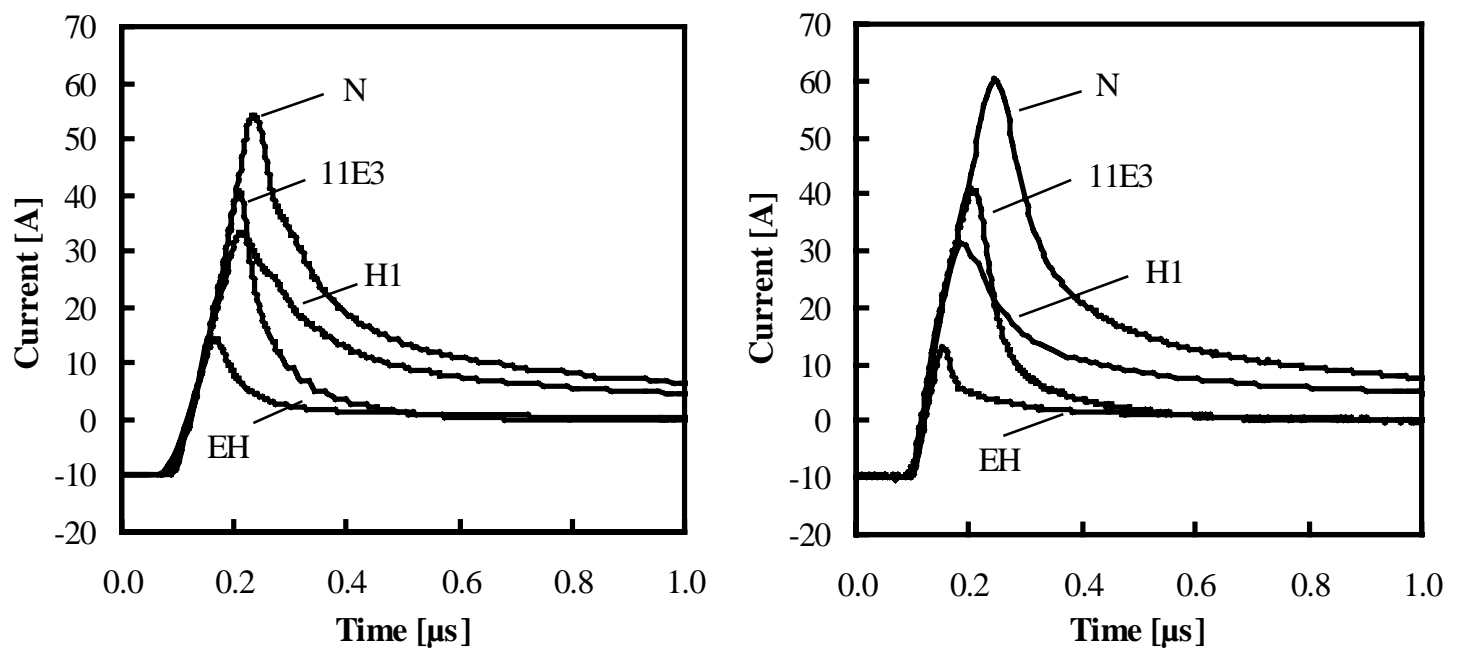

Figure 5: Comparison of reverse recovery current for different samples in simulation (left) and experiment (right)

in simulation and lead to satisfying results.

For optimization purposes of irradiated power devices , the use of device simulation now offers enhanced possibilities since both, stationary as well as dynamic behavior, are correctly calculated by the simulation tool.

Another possibility offered by the use of device simulation is to look inside the device. Under normal operating conditions, a sinusoidal current is often chopped at lower than rated current. In fact, low current is a critical condition for the reverse recovery of a freewheeling diode since the number of stored carriers is significantly reduced which often leads to a snap-of in the reverse current. This results in overvoltages and/or oscillations due to parasitic inductances. Figure 7 compares the reverse recovery of the snappy sample $11 \mathrm{E} 3$ (left side) and the soft sample EH (right side) at low current density of app. $10 \%$ of the rated current density.

The explanation for the different behavior of this samples is shown in figure 8 . There, the hole carrier densities of the snappy and the soft freewheeling diode are shown. In the snappy device, the carriers at the $\mathrm{nn}^{+}$junction are already strongly reduced while at the same time there are still carriers at the pn-junctions side. To prevent snap-of during reverse recovery the number of holes at the pn-junction should be slightly lower than the number of electrons at the $\mathrm{nn}^{+}$-junction as shown on the right side of figure 8 . Here, even at the end of the reverse recovery process a small number of carriers is found which results in a soft current tail. Thus, simulations may be used for the explanation of device behavior, resulting in a better understanding of the device.

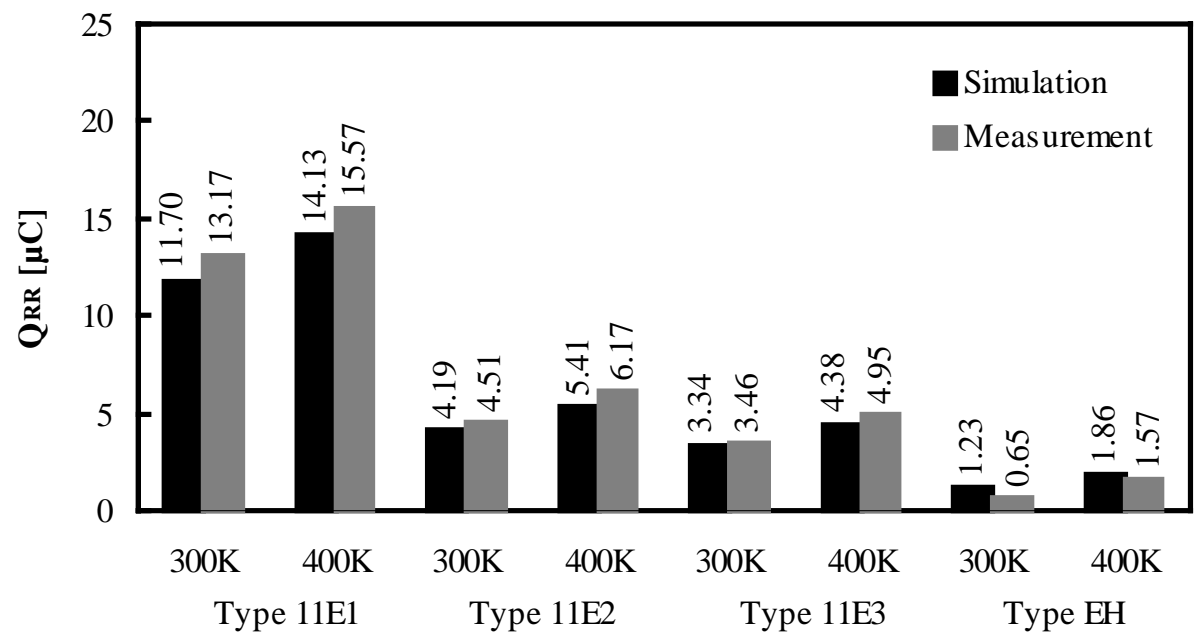

Figure 6: Stored charge for different samples at $V_{R}=250 V, I_{F}=10 A$ and di/dt=500A/ $\mu \mathrm{s}$ 


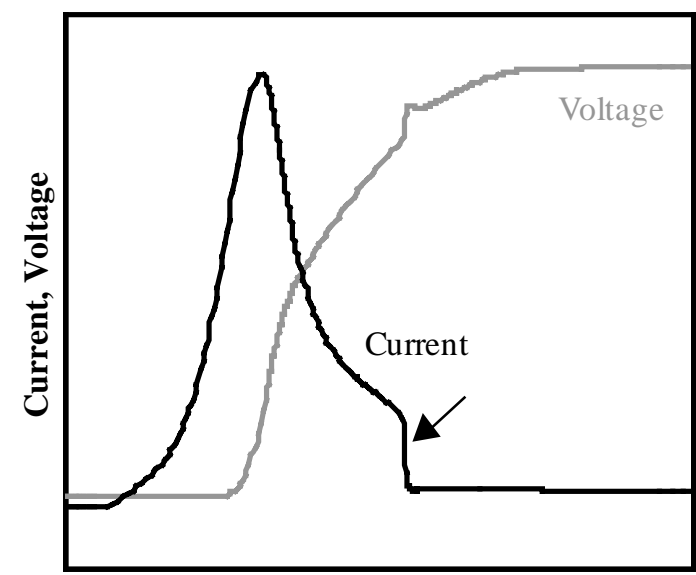

Time

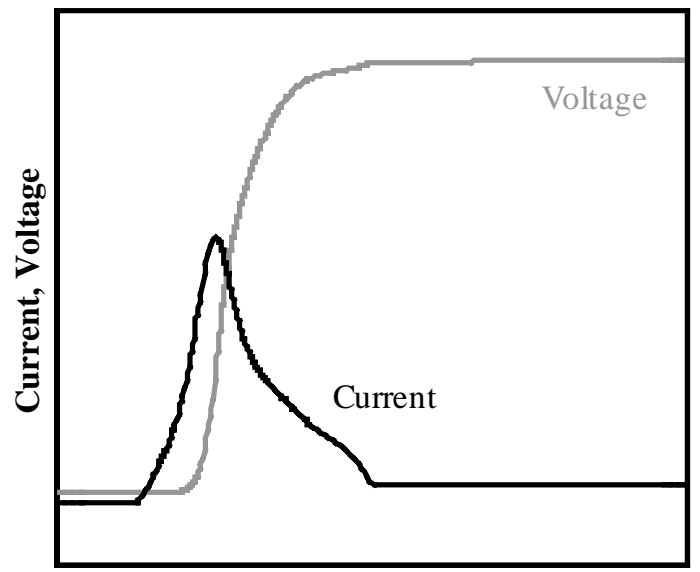

Time

Figure 7: Reverse recovery characteristics of a snappy and a soft freewheeling diode ar low current density

\section{CONCLUSION}

The use of an extended recombination model including full trap dynamics for the simulation of radiated devices results in a good accordance between measurements and simulations. The advanced model also considers the charging processes of the recombination centers, thus allowing correct transient simulations of the space charge region. The introduction of several recombination centers with different properties into simulation further allows the correct description of recombination processes under different conditions such as high- or low-injection or carrier generation in a space charge region, since they are controlled by different traps. The parameters that were used for the simulations explain the temperature dependencies of stationary and dynamical characteristics. Therefore, simulations give the possibility to look inside the device and to analyze and improve device characteristics. Thus, simulation may now be used for development and optimization of radiated devices.

\section{ACKNOWLEDGEMENTS}

The authors wish to thank the scientists, especially Dr. Nürnberg and Prof. Gajewski, from the Weierstrass Institute for Applied Analysis and Stochastics in Berlin, who developed the device simulator TeSCA. In addition the authors are grateful for their support and the addition of new features and algorithms into the simulation system.

The Authors wishes to thank also Ms. Pellkofer and Mr. Umland for their support in sample preparation and measurement assistance. We also wishes to thank Prof.

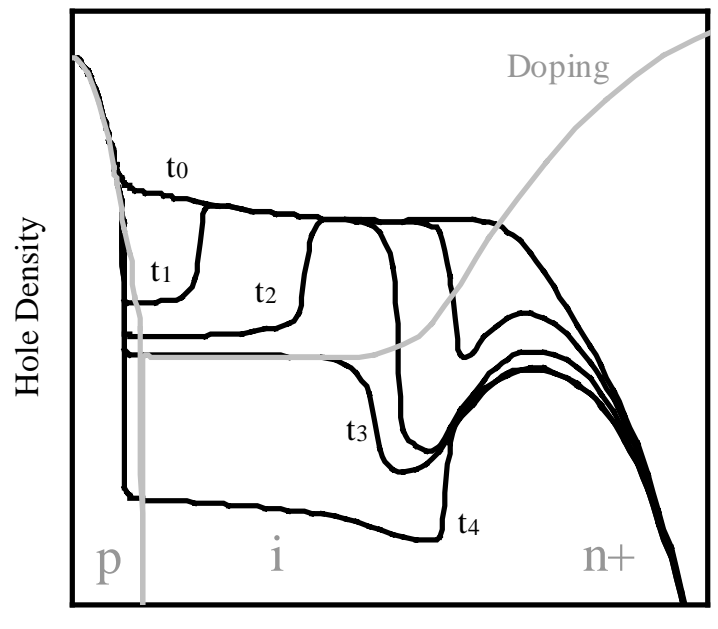

Depth

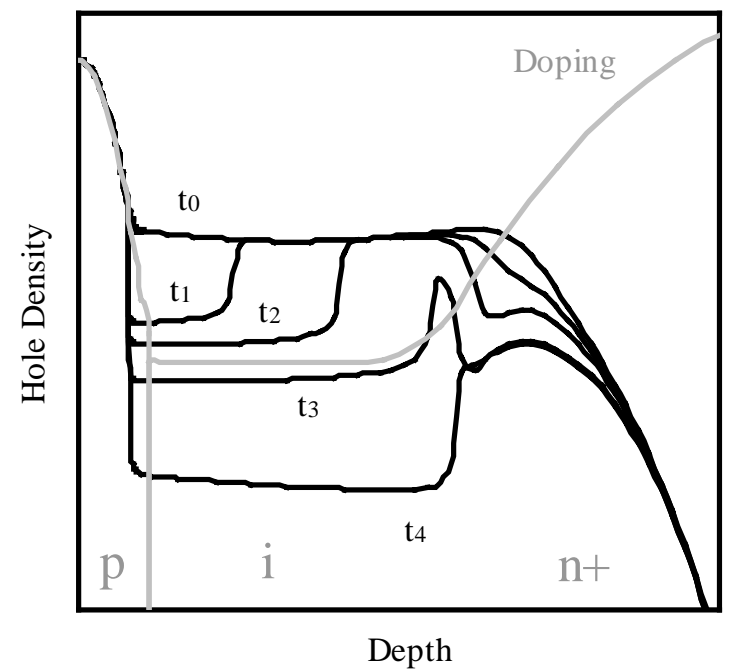

Figure 8: Carrier distribution during turn-off in a snappy (left) and soft (right) diode sample 
Wagemann at the Technical University of Berlin for the possibility to perform DLTS measurements.

This work is supported by grants of the Deutsche Forschungsgemeinschaft.

\section{REFERENCES}

1 J.Lutz. Axial Recombination Center Technology for Freewheeling Diodes, Proceedings of the 7th EPE, Trondheim, (1997)

2 J.Lutz, U.Scheuermann. Advantages of the New Controlled Axial Lifetime Diode, Proceedings of the 28th PCIM (1994)

3 D.V.Lang. Deep-level transient spectroscopy: A new method to characterize traps in semiconductors, Journal of Applied Physics, Vol.45, No.7, pp.3023-3032, July 1974

4 S.R.Lederhandler, L.J.Giacoletto. Measurement of Minority Carrier Lifetime and Surface Effects in Junction Devices, Proc. IRE, pp.477-483, April 1955

5 R.Siemieniec, D.Schipanski, W.Südkamp, J.Lutz. Simulation and Experimental Results of Irradiated Power Diodes, Proc. EPE 1999, Lausanne

6 R.Siemieniec, W.Südkamp, J.Lutz. Determination of Parameters of Radiation Induced Traps in Silicon, accepted for publication in Solid-State Electronics

7 Gajewski et al. TeSCA-Handbuch, WIAS Berlin, 1994

8 J.Vobecky, P.Hazdra, V.Zahlava. OCVD Lifetime of Ion Irradiated $P-i-N$ Diodes, Proceedings of the ISPS'98, pp.47-52, Praha, 1998

\section{APPENDIX - SYMBOLS}

hole concentration electron concentration generation rate recombination rate acceptor density donor density ionized acceptor density ionized donor density acceptor trap density ionized acceptor trap density donor trap density ionized donor trap density fraction of occupied acceptor traps fraction of occupied donor traps capture rate of acceptor trap for electrons capture rate of acceptor trap for holes emission rate of acceptor trap for electrons emission rate of acceptor trap for holes capture rate of donor trap for electrons capture rate of donor trap for holes emission rate of donor trap for electrons emission rate of donor trap for holes elemental charge time electron current density hole current density 